\title{
Demokratisierung von Technik ohne Wirtschaftsdemokratie?
}

\author{
TA und die Frage demokratischer Verhältnisse in der Wirtschaft
}

\author{
Philipp Frey, Institut für Technikfolgenabschätzung und Systemanalyse (ITAS), Karlsruher Institut für Technologie (KIT), Karlstr. 11, 76133 Karlsruhe \\ (philipp.frey@kit.edu) (10 https://orcid.org/0000-0002-7780-9081 \\ Christoph Schneider, Institut für Technikfolgenabschätzung und Systemanalyse (ITAS), Karlsruher Institut für Technologie (KIT) \\ (christoph.schneider@kit.edu) (1) https://orcid.org/0000-0003-1516-158X \\ Christian Wadephul, Institut für Technikfolgenabschätzung und Systemanalyse (ITAS), Karlsruher Institut für Technologie (KIT) \\ (christian.wadephul@kit.edu) (10 https://orcid.org/0000-0002-0327-1733
}

Der vorliegende Beitrag diskutiert vor dem Hintergrund der gegenwärtigen Dominanz ökonomischer Akteure in der Entwicklung und dem Einsatz von Technik, inwiefern das Einstehen der Technikfolgenabschätzung (TA) für eine demokratische Gestaltung des technischen Wandels durch Wirtschaftsdemokratie erweitert werden könnte. Anschließend werden einige Grundlinien wirtschaftsdemokratischer Theorieansätze der Gegenwart präsentiert, um abschließend danach zu fragen, wie diese die Arbeit der TA konkret informieren und orientieren könnten.

\section{Democratizing technology without democratizing the economy? TA and the question of democratic relations in the economic sphere}

In light of the current dominance of economic actors in the development and application of technology, this paper discusses whether the commitment of technology assessment (TA) to democratizing technological change could or should be extended to include a commitment to economic democracy. Subsequently, we present some principles of contemporary theoretical approaches to economic democracy and finally ask how these could inform and orient the work of TA in concrete terms.

Keywords: economic democracy, political economy, technology assessment, digitalization

\section{Einleitung}

Technikfolgen lassen sich häufig ohne Reflexion der ökonomischen Bedingungen von Technikentwicklung und -einsatz kaum verstehen. Auch die Bedrohung und Erosion demokratischer Strukturen steht in Zusammenhang mit der ökonomischen Verfasstheit spätkapitalistischer Gesellschaften: Während Digitalisierung und Klimakrise die Grenzen und Funktionen von Technik verschieben und erweitern, stehen weltweit demokratische Institutionen unter Druck. Autoritäre Kräfte, unregulierte Konzerne, aber auch sinkendes Vertrauen in die Institutionen liberaler Demokratie tragen zunehmend zu einer Verengung demokratischer Horizonte bei. Technikfolgenabschätzung (TA) bleibt von solchen Dynamiken nicht unberührt. Steht doch zum einen die komplexe Beziehung zwischen technischem und gesellschaftlichem Wandel im Zentrum der TA. Zum anderen ist die TA auf unterschiedliche Weise der Demokratie, der wissenschaftlichen Beratung politischer Akteure, der Ermöglichung von Alternativen verpflichtet. Zudem verändern sich die Verhältnisse zwischen Wissenschaft, Politik, Wirtschaft und Gesellschaft massiv (Maasen et al. 2020) - was Fragen zur Rolle und Position von TA und ihren normativen und epistemischen Orientierungen aufwirft.

Wir argumentieren, dass die die TA mittragende Idee einer Demokratisierung des technischen Wandels so verstanden werden kann und sollte, dass sie auch Überlegungen zur Demokratisierung wirtschaftlicher Prozesse umfasst. Dies diskutieren wir anhand einer Reflexion der massiv angewachsenen technischen Macht im gegenwärtigen Kapitalismus und der Krise (neo-)liberaler Demokratie. Das Paper skizziert hierauf aufbauend Grundlinien wirtschaftsdemokratischer Theorieansätze und versucht, sie für TA-Perspektiven fruchtbar zu machen. Abschließend diskutieren wir mögliche Implikationen einer solchen Weitung des Demokratieverständnisses für die wissenschaftliche Praxis der TA.
This is an article distributed under the terms of the Creative Commons Attribution License CCBY 4.0 (https://creativecommons.org/licenses/by/4.0/) https://doi.org/10.14512/tatup.29.3.30

Submitted: 08. 06.2020. Peer reviewed. Accepted: 21.10.2020 


\section{Technik und Macht im neoliberalen Kapitalismus}

Dass die Entwicklung und Nutzung von Technologie ein machtdurchsetzter Prozess ist, dürfte den meisten Leser*innen selbstverständlich sein. Wer über technische Dinge und Infrastrukturen entscheidet, sie gestaltet, produziert, verkauft oder reguliert, übt Macht aus (Hubig 2015). Wir wollen diese allgemeine Einsicht aber in eine Gegenwartsdiagnose einbetten, die uns die Frage nach der Demokratisierung von Technik und Wirtschaft geradezu aufdrängt.

Seit den späten 1970er-Jahren beseelt das 100 Jahre alte Dogma der angeblich idealen Regulierung durch die ,unsichtbare Hand des Marktes ' auch innovations- wie wirtschaftspolitische staatliche Reformprojekte, welche Märkte von politischer Gestaltung zu befreien suchen (Stichwort De-Regulierung). Zum anderen wird in dieser (neo-)liberalen Logik Staatsmacht dazu verwandt, vormals nicht-marktförmig organisierte Bereiche der Gesellschaft privatwirtschaftlicher Logik zuzuführen (Stichwort Privatisierung). Durch die einseitige Betonung der ,unsichtbaren Hand des Marktes " "wird hierbei explizit die Forderung nach einer undemokratischen Organisation der Ökonomie erhoben' ${ }^{1}$. Das Beharren auf der Trennung von Wirtschaft und Demokratie scheint dabei konsequenterweise nicht unvereinbar mit robuster Einflussnahme wirtschaftlicher Akteure auf die Politik, etwa im Interesse von Deregulierung. Diese Einflussnahme etwa durch den Lobbyismus der Automobilindustrie, durch Desinformationskampagnen der Ölindustrie oder digitale Filterun-
(Tyfield et al. 2017). Zum anderen wurde Technik und technisches Wissen (privatisiert durch Patente) zu dem entscheidenden Faktor im neoliberalen Kapitalismus. Obwohl die HightechWissensökonomie des Digitalzeitalters verhältnismäßig wenige Menschen beschäftigt, steht sie, neben den durchdigitalisierten Finanzmärkten, im Zentrum des gegenwärtigen Kapitalismus was durch die Coronakrise nur bekräftigt wurde. Nicht nur zählen die monopolistischen Digitalkonzerne zu den Firmen mit den weltweit höchsten Marktkapitalisierungen, sie stehen zudem oftmals im Zentrum soziotechnischer Systeme, die für Millionen, wenn nicht gar Milliarden Menschen immer stärker den Alltag prägen. Hiermit gehen historisch nie dagewesene Kontroll- und Überwachungsmöglichkeiten von Menschen sowie exzessive ökonomische und politische Macht einher (Sadowski 2020). Diese geraten dabei in Konflikt mit dem Anspruch demokratischer Souveränität und Gestaltbarkeit und betreffen das öffentliche wie private Leben: Das neoliberale Profitstreben hat sich in den Großteil der soziotechnischen Infrastrukturen unserer Gesellschaften eingenistet (Srnicek und Williams 2016).

Doch hierdurch ist Technik auch zu einem wesentlichen Politikum unserer Zeit geworden. Neben den umfassenden Umbrüchen der Digitalisierung formieren sich in der Klimakrise breite Auseinandersetzungen um Infrastrukturen und deren Veränderung, dringen Technikvisionen in politische Diskurse ein, werden Medikamente gegen COVID-19 im Wettbewerb hinter verschlossenen Türen erforscht und menschliche Tätigkeit zunehmend maschinisiert und technisch ersetzt. Technik vermittelt Macht - und das komplexer und umfassender denn je zuvor. Auf-

\section{Wie lassen sich demokratische Ansprüche auch in der ökonomischen Sphäre für die TA fruchtbar machen?}

gen des öffentlichen Diskurses durch Plattformkonzerne - droht im Gegenzug nicht nur die öffentliche Debatte zu vergiften, sondern auch demokratische Institutionen zu unterminieren.

In Bezug auf Technik sind zwei Entwicklungen der neoliberalen Ära von besonderer Bedeutung. Zum einen verschob sich die Macht über Forschung und Entwicklung von Technologie zunehmend in den privatwirtschaftlichen Bereich, zu Großkonzernen, Finanzinvestoren und Start-Ups. Teil dieser Privatisierungsbewegung technischer Entwicklung sind auch die neuen Governance-Formen des Wissenschaftsbetriebs, welche auf Drittmittel-Abhängigkeit (auch von Firmen) setzen, Output in Form von Patenten und Gründungen belohnen und wirtschaftliche Innovation als neue Aufgabe für Universitäten definieren

1 Es liegen zahlreiche Analysen des Neoliberalismus vor. Die inhärent antidemokratische Dimension neoliberaler Ideologie und Politik verdeutlicht besonders Slobodian (2018) grund der enormen Bedeutung der Ökonomie für Technikentwicklung und -einsatz steht die TA vor einem Problem: Fühlt sie sich einem demokratischen Gestaltungsanspruch verpflichtet, so wird dieser in einem Schlüsselbereich negiert.

Dabei muss sich die TA vor dem Hintergrund der gegenwärtigen gesellschaftlichen Herausforderungen auch Fragen jenseits der „Nebenfolgen“ von Technik stellen. Immer mehr sind es auch die durch mächtige Akteure beabsichtigten Folgen im $\mathrm{Zu}$ sammenhang mit neuer Technik - etwa exzessives Wirtschaftswachstum, aber auch ,Disruption“ ganzer Branchen - welche uns vor komplexe soziotechnische Probleme stellen, die nicht nur privatwirtschaftliche, sondern immer auch ökologische und letztlich politische Dimensionen haben. Deshalb sollte die Suche der TA nach „Alternativen“ (Dobroć et al. 2018) verstärkt auch Alternativen der gesellschaftlichen und wirtschaftlichen Einbettung von Technik in den Blick nehmen und auf ein Primat des demokratischen Diskurses und rationaler Abwägung be- 
stehen, wo derzeit die bornierte Fixierung auf Profitmaximierung dominiert (Krüger und Frey 2020). Wie aber lassen sich demokratische Ansprüche auch in der ökonomischen Sphäre konzeptionell fassen und für die TA fruchtbar machen?

\section{Demokratische Gestaltung techno-ökonomischer Prozesse}

Dass Technik Macht vermittelt, Ungleichheiten formt und tief mit ökonomischen Verhältnissen verbunden ist, ist keine neue Einsicht. Marx und Engels stellten Mitte des 19. Jahrhunderts den Besitz von Produktionsmitteln, also Technik, gar ins Zentrum ihrer Analyse von Klassenunterschieden: Die Bourgeoisie besitzt die neue Technik, die Arbeiterklasse muss die eigene Lebenszeit für Einkommen verkaufen. Politisch leiteten sie eine klassische Idee der Wirtschaftsdemokratie hiervon ab: Es sei notwendig die Produktionsmittel zu vergesellschaften damit die Menschen über diese entscheiden und sie frei und gleich nutzen können. Übersetzt in heutige Sprache geht es hier um eine radikale Demokratisierung von Technik. Doch was unter Wirtschaftsdemokratie genau verstanden werden soll, ist selbst unter ihren Vertreter*innen teilweise heftig umstritten. Zwar können wir die Geschichte wirtschaftsdemokratischen Denkens hier nicht erschöpfend darstellen (siehe hierfür Demirović 2018), wir wollen aber dennoch versuchen, einige zentrale Momente von fortdauernder Relevanz zu diskutieren:

Für lange Zeit prägend für die deutschsprachige Debatte war das Programm zur Demokratisierung der Wirtschaft, das eine Kommission unter Leitung von Fritz Naphtali im Auftrag des Allgemeinen Deutschen Gewerkschaftsbundes erarbeitete und das 1928 verabschiedet wurde. In der frühen Bundesrepublik konnte der Deutsche Gewerkschaftsbund mit seinem Münch-
S. 18). Wirtschaftsdemokratie wird dabei als eine „Ergänzung zur politischen Demokratie“ sowie „eine Form der wirtschaftlichen Verfassung“ (ebd.) verstanden. Naphtalis Wirtschaftsdemokratie-Verständnis liegt damit die Vorstellung einer fundamentalen Neuordnung von Wirtschaft und Gesellschaft zugrunde.

Wir schließen uns im Folgenden der für die TA handhabbareren Definition von Fritz Vilmar an. Nach diesem ist Wirtschaftsdemokratie der „Inbegriff aller ökonomischen Strukturen und Verfahren, durch die an die Stelle autokratischer Entscheidungen demokratische treten, die durch die Partizipation der ökonomisch Betroffenen und/oder des demokratischen Staates legitimiert sind“" (Vilmar 1975, S. 28). Da wir es im gegenwärtigen digitalisierten Kapitalismus mit in alle Lebensbereiche verzweigten ökonomischen Strukturen zu tun haben, muss der Horizont von Wirtschaftsdemokratie über die etablierten Formen gewerkschaftlicher Mitbestimmung in Betrieben hinausgedacht und zeitgemäß rekonzeptualisiert werden. Die anspruchsvolle Aufgabe einer radikalen Wirtschaftsdemokratie besteht darin, eine grundsätzliche „Herstellung des Primats demokratischer Politik über die Ökonomie“" und ,eine gerechtere Verteilung des gesellschaftlichen Reichtums“, zu welchem auch technische Potenziale zählen, zu erreichen (Martens und Scholz 2010, S.31) - ein Auftrag, der auf einzelbetrieblicher Ebene nur schwer umsetzbar scheint. Die TA mit ihrer Sensibilität für die Komplexität soziotechnischer Gemengelagen scheint uns gut aufgestellt, hier mit Blick auf Technik aufzuklären und mitzugestalten.

Wirtschaftsdemokratie verlangt, dass ökonomische Machtund Eigentumsstrukturen demokratisiert werden. Schaut man, wer von den ökonomischen Entscheidungen und neuen Technologien am stärksten betroffen ist, kommen neben den Arbeitnehmer*innen weitere Individuen und Institutionen in den Blick: Nutzer*innen, Stadtbewohner*innen, Bürger*innen, Patient*innen, Schulen, Krankenhäuser, Kommunen und mehr. Die Frage,

\section{TA sollte ihre etablierte Rolle als neutrale Vermittlerin durch wirtschaftsdemokratische Perspektiven erweitern.}

ner Grundsatzprogramm für eine Neuordnung der Wirtschaft von 1949 an diese wirtschaftsdemokratische Ideen aus der Zwischenkriegszeit anschließen. Grundlage für eine Demokratisierung der Wirtschaft ist nach Naphtali die Institutionalisierung der politischen Demokratie: „Die Idee der Wirtschaftsdemokratie kann nur dann in einem Lande lebendig werden, wenn die Arbeiterschaft dieses Landes schon bestimmte Erfahrungen in der politischen Demokratie hat." (Naphtali 1928/1966, S. 13) Angesichts der wirtschaftlichen Autokratie kapitalistischer Wirtschaften und ihrer Tendenz zur Kommodifizierung lebenswichtiger Gesellschaftsbereiche zum Leidwesen der Bevölkerung forderte Naphtali einen „Vormarsch der öffentlichen Wirtschaft“ (ebd., wie man diese in Technisierungsprojekten demokratisch inkludiert - und zwar über rein epistemische Überlegungen und unverbindliche Ideenworkshops hinaus - ist nicht trivial. Nicht nur staatlich getragene Modelle, sondern genauso genossenschaftliche und an Gemeingütern (commons) orientierte Ansätze (Scholz und Schneider 2017) können je unterschiedlich einen Ausgleich zur ökonomischen und politischen Dominanz von Privateigentum im Neoliberalismus schaffen.

Angesichts von Corona- und Klimakrise ist es zunehmend wichtig, Wirtschaftsdemokratie nicht nur als Chance, sondern sogar als Bedingung für eine zügige und gerechte Transformation soziotechnischer Infrastrukturen zu sehen (Urban 2019). Eine 
Vertiefung wirtschaftsdemokratischer Strukturen könnte die Gestaltung von Technik und die Diskussion über Technikfolgen radikal verändern: Die „fremde“ Technik könnte durch eine Stärkung der Gestaltungsmacht der von ihr Betroffenen zu ,unserer" Technik werden. Damit könnte auch ein wichtiger Beitrag geleistet werden, um die in der Wahrnehmung vieler Menschen auf einen Wahlakt alle paar Jahre verkümmerte Idee der Demokratie zu revitalisieren. An die Stelle empfundener Ohnmacht gerade im Kontext der Digitalisierung - könnte so durch eine Reorganisation der Sphäre unserer materiellen Reproduktion das Erproben und Einüben demokratischer Prozesse im Alltag treten. Dies könnte nicht zuletzt auch helfen, Tendenzen in Richtung Autoritarismus entgegenzutreten (Hilmer et al. 2017). Oder wie Oskar Negt einmal pointiert formulierte: ,Keine Zukunft der Demokratie ohne Wirtschaftsdemokratie“ (Negt 2011).

\section{TA als demokratisierende Gesellschaftsberatung stärken}

Auch wenn , die Wirtschaft' wiederholt Gegenstand von Debatten innerhalb der TA war (Grunwald 2001), steht eine systematische Beschäftigung mit der Veränderbarkeit der technisierten Wirtschaft, eine zentrale gesellschaftliche Machtfrage unserer Zeit, noch aus. Die TA sollte diese Frage aus unserer Sicht nicht ignorieren, gerade weil sie sich der Demokratisierung unserer gesellschaftlichen Verhältnisse verpflichtet fühlt. Aber was würde eine solche Beschäftigung implizieren und wie legitimierte sich eine solche Form der TA?

Wirtschaftsdemokratie ist verbunden mit politischen Traditionslinien, die oft als links oder progressiv bezeichnet werden. Geht es doch um die Ausweitung demokratischer Freiheits-, Gleichheits- und Mitbestimmungsrechte, also um zentrale Momente sozialen Fortschritts. Vermutlich ist das auch ein Grund, weshalb Wirtschaftsdemokratie bislang kaum in der TA verhandelt wurde. Denn im wohl am weitesten verbreiteten Leitbild der TA, in ihrer klassischen Institutionalisierung als parlamentarische TA, soll sie als neutrale Vermittlerin zwischen verschiedenen gesellschaftlichen Positionen agieren. Eine Positionierung, die als Parteinahme für eine bestimmte politische Tradition verstanden werden kann, ist hier kontraproduktiv - wenngleich auch die Institutionalisierung der parlamentarische TA als progressive Entwicklung gelesen werden kann, in der sich gegenüber einem früheren Status Quo durch die Stärkung der Legislative eine Ausweitung der Demokratie durchgesetzt hat (Delvenne und Parotte 2019).

TA erschöpft sich aber nicht in der parlamentarischen TA. Vielmehr hat sie sich in einer wachsenden Community ausdifferenziert und tritt auch als Gesellschaftsberaterin und Teilnehmerin im öffentlichen Diskurs auf. Ein Diskurs, der - das wird gegenwärtig deutlich - konstitutiv ein politischer ist (Schröder 2019), gerade in einer Gesellschaft, die Technik und Wissenschaft zunehmend als Politikum begreift (Maasen et al. 2020). Im Folgenden greifen wir die Frage auf, wie TA in diesem Dis- kurs neben ihrer etablierten Rolle als neutrale Vermittlerin ihre jüngere, transdisziplinäre Rolle als Transformationsagentin - etwa in den Bereichen Responsible Research and Innovation (RRI) oder Nachhaltigkeitsforschung - durch wirtschaftsdemokratische Perspektiven erweitern kann. So wurde insbesondere in der ,transformativen Forschung“ (Schneidewind 2018) in letzter Zeit intensiv diskutiert und erprobt, wie Forschung, die auf systemische Veränderungen gerichtet ist, praktisch aussehen kann und welche Legitimationsherausforderungen und -chancen sich für sie ergeben. Hieran anschließend könnten innerhalb der TA-Community im Sinne eines theoretischen wie methodischen Pluralismus vermehrt auch Diskussionen und Projekte zu Wirtschaftsdemokratie ihren Raum finden und so eine weitere Ausdifferenzierung der TA ermöglicht werden.

\section{Demokratisierung von Innovationsregimen}

Zunächst scheinen uns insbesondere die Überlegungen von Schombergs (2013) anschlussfähig, der seine „Vision for Responsible Research and Innovation" abgrenzt von rein nach Marktlogiken organisierter Innovation. Statt Wettbewerb, Angebot und Nachfrage sollen demokratisch legitimierte Grundwerte Innovationen anleiten und verantwortungsvoll gestalten helfen. Während die RRI-Diskussion bislang mit epistemischem Fokus verlief und fragte, wie Verantwortung in Innovationsprozessen mitgedacht werden kann, steht eine breite Auseinandersetzung dazu, wie RRI durch politisch-ökonomische Veränderungen auch realisiert werden könnte, noch aus (Tyfield et al. 2017). Zentral wäre hier insbesondere die Frage, wie demokratische Prozesse im Bereich Wissenschaft und Innovation organisiert und institutionalisiert werden können. Neben der Frage nach $\mathrm{Ge}$ schäftsmodellen und Wirtschaftsregulierungen, die diese überhaupt ermöglichten, wäre dann auch eine stärkere Befassung mit alternativen Innovationsansätzen notwendig: Open Innovation, Open Science, soziale Innovationen, Vertiefung der Demokratie in der Forschungsförderung, Digitalgenossenschaften und mehr.

\section{Gemeinwohlorientierung der Wissenschaft stärken}

Parallel hierzu läuft die Frage, wie sich eine TA, die sich für die Vertiefung der Demokratie einsetzt, in ihrer Praxis legitimiert. Eine verstärkte Betonung der partizipativen und inklusiven Dimension der TA ist hier eine Möglichkeit. Zunächst wäre die Frage, ob wirtschaftsdemokratische Perspektiven - die es ja durchaus im gesellschaftlichen Diskurs gibt - bislang ausreichend in partizipativen Formaten der TA wiedergespiegelt werden. Im Forschungsfeld Arbeit und Technik könnten vermehrt die Bedarfe und Interessen von Belegschaften und Betriebs- und Personalräten sowie den Gewerkschaften eingebunden werden. Bei Fragen von gesellschaftlicher Veränderung verdient das Engagement von TA-Wissenschaftler*innen in sozialen Bewegungen und zivilgesellschaftlichen Organisationen sowie die direkte Arbeit mit Bürger*innen mehr Aufmerksamkeit. Hier können Schnittstellen zwischen Wissenschaft, Gesellschaft und Zukunftsgestaltung gelebt werden. Es stellt sich also nicht nur die Frage, wie Bürger*innen an wissenschaftlichen Prozessen par- 
tizipieren können, sondern auch, wie umgekehrt TA an öffentlichen Auseinandersetzungen für mehr Demokratie teilhaben kann. TA könnte sich hier einbringen, um Themen- und Akteurskonstellationen zu weiten und durch ihren vermittelnden Ethos politische Gräben überwinden zu helfen.

Engagement für eine Demokratisierung gesellschaftlicher Verhältnisse und die damit einhergehende vermehrte Zusammenarbeit mit zivilgesellschaftlichen Akteuren evoziert allerdings auch eine Ressourcenfrage: Es benötigt Zeit und Geld, die an anderer Stelle fehlen würden - während es gleichzeitig nur begrenzte Möglichkeiten zur Mittelakquise eröffnen dürfte. Hier wäre die TA aus unserer Sicht insbesondere dazu aufgerufen, ihr soziales Kapital auch öffentlich für die langfristige Sicherung gemeinwohlorientierter Forschung in die Waagschale zu werfen. Nicht nur ist das projektförmige, drittmittelabhängige Finanzierungssystem wissenschaftlicher Forschung eine schmerzhafte Zumutung für die betroffenen Wissenschaftler*innen; sie verträgt sich auch nur schlecht mit der Stärkung langfristiger demokratischer Prozesse, die auch verlässliche Ansprechpartner*innen voraussetzen.

\section{Wirtschaftsdemokratische Realexperimente}

Abseits allgemeiner Erwägungen scheinen sich uns auch konkrete Leuchtturmprojekte einer solchen (wirtschafts-)demokratischen TA anzubieten. So hat sich in den letzten Jahren der Reallaboransatz in der TA weiter etabliert (Quartier Zukunft et al. 2020). In Reallaboren könnten TA-Wissenschaftler*innen als Initiator*innen und Mediator*innen demokratischer Aushandlungen über die Zukunft von Technikentwicklung und -nutzung in der ökonomischen Sphäre fungieren. Wie ließen sich Innovationen und Investitionen demokratisieren? Welche Technologien passen $\mathrm{zu}$ den Bedürfnissen der Beschäftigten und Nutzer*innen? Welche Möglichkeiten demokratischer Organisation, wie etwa Genossenschaften, gibt es in der wirtschaftli- loten (Kulke und Wadephul 2020; Jochum und Schaupp 2019)? Solche Fragen könnten unter Einbezug verschiedenster Stakeholder-Gruppen wissenschaftlich informiert, gerne auch kontrovers, diskutiert und praktisch ausprobiert werden.

\section{Offene Fragen für die weitere Debatte}

Mit dem hier dargestellten Fokus auf Wirtschaftsdemokratie erweitert sich die Perspektive auf das, was unter Demokratie verstanden wird: Der Geltungsbereich demokratischer Werte wird auf die Wirtschaft, einen Schlüsselbereich gesellschaftlicher Innovation, ausgedehnt. Wäre eine solche Erweiterung ihres Demokratieverständnisses nicht ein weiterer konsequenter Schritt für eine TA, die sich Inklusion, Partizipation und Deliberation verschrieben hat und diese Werte auch in ihrer Praxis zu realisieren versucht?

Mit diesem erweiterten Demokratieverständnis würde für die TA neben die Rolle der neutralen Vermittlerin (innerhalb etablierter demokratischer Strukturen) auch die Rolle einer Mitgestalterin öffentlicher Zukunftsdebatten (mit dem Ziel einer Vertiefung der Demokratie) annehmen. Unabhängig von den konkreten Antworten, kommt TA in diesem (transformativen) Selbstverständnis stärker die Rolle zu, alternative soziotechnische Zukünfte denk- und diskutierbar zu machen (Delvenne und Parotte 2019; Schröder 2019). Solche ,konkreten Utopien“ sollten neue Technologien und demokratische Neuerungen zusammendenken (Frey und Schaupp 2020), sodass komplexe Verflechtungen normativer Orientierungen und technischer Potenziale greifbar und verhandelbar werden.

Zur inhaltlichen Schärfung (Wirtschaftsdemokratie) träte dann auch die experimentelle Suche nach den passenden Praxisformen (Demokratisierung), in denen die TA als Community auch lernen könnte, in unterschiedlichen Rollen zu agieren und

\section{Wie kann die TA ihre Teilhabe an öffentlichen Auseinandersetzungen für mehr Demokratie stärken?}

chen Sphäre? Wie kann und sollte die Digitalisierungsdividende umverteilt werden? Wie (wieviel, mit was für Technik, mit welchem Ziel) wollen wir in Zukunft vor dem Hintergrund des technologischen Wandels arbeiten? Wie ließe sich der ohnehin anstehende Umbau grundlegender soziotechnischer Infrastrukturen mit einem neuen demokratischen Aufbruch verbinden? Und welchen Beitrag könnte eine Erneuerung von Planungstheorien wie der Kybernetik kombiniert mit den Potenzialen des maschinellen Lernens für die Entwicklung einer digital-deliberativen Wirtschaftsdemokratie leisten? Wie könnte eine dergestalt institutionell wie technisch modernisierte Demokratie dabei helfen, möglichst inklusiv Möglichkeiten einer bestmöglichen Bedürfnisbefriedigung innerhalb der planetarischen Schranken auszu- das Spannungsfeld zwischen Neutralität und Engagement kontextsensitiv zu navigieren. Natürlich verbleibt ein großer Diskussionsbedarf: Können und dürfen TA Projekte transformativ zu Wirtschaftsdemokratie forschen, obwohl das Leitbild hierzu weniger breit in der Gesellschaft angekommen ist, als das der (ökologischen) Nachhaltigkeit? Welche Verbindungen lassen sich hier finden? Welche Bündnispartner sind hier denkbar, ohne reine $\mathrm{Ge}$ werkschafts-TA zu werden? Wie kann ein produktiver, demokratieorientierter Umgang mit der Normativität solcher Forschungsperspektiven aussehen, der möglichst die Legitimation anderer Formen von TA nicht unterminiert? Obwohl es hier, wie gezeigt, Anschlüsse gibt, bleiben noch viele Fragen offen. Aber schließlich ist ja auch die Demokratie selbst ein unfertiges Projekt. 


\section{Literatur}

Delvenne, Pierre; Parotte, Céline (2019): Breaking the myth of neutrality. Technology assessment has politics, technology assessment as politics. In: Technological Forecasting and Social Change 139, S. 64-72. D0I: 10.1016/ j.techfore.2018.06.026

Demirović, Alex (Hg.) (2018): Wirtschaftsdemokratie neu denken. Münster: Westfälisches Dampfboot.

Dobroć, Paulina; Bettina-Johanna Krings; Christoph Schneider; Nele Wulf (2018): Alternativen als Programm. In: TATuP - Zeitschrift für Technikfolgenabschätzung in Theorie und Praxis 27 (1), S. 28-33. D0I: 10.14512/tatup.27.1.28.

Frey, Philipp; Schaupp, Simon (2020): Futures of digital industry. Techno-managerial or techno-political utopia? In: Behemoth - A Journal on Civilization 13 (1), S. 98-108. DOI: 10.6094/behemoth.2020.13.1.1039.

Grunwald, Armin (2001): Technikfolgenabschätzung und Industrie. In: TATuP Zeitschrift für Technikfolgenabschätzung in Theorie und Praxis 10 (2), S. 3-6. DOI: 10.14512/tatup.10.2.3.

Hilmer, Richard; Kohlrausch, Bettina; Müller-Hilmer, Rita; Gagné, Jérémie (2017): Einstellung und soziale Lebenslage. Eine Spurensuche nach Gründen für rechtspopulistische Orientierung, auch unter Gewerkschaftsmitgliedern (Working Paper Forschungsförderung 044). Düsseldorf: Hans-Böckler-Stiftung. Hubig, Christoph (2015): Die Kunst des Möglichen III. Grundlinien einer dialektischen Philosophie der Technik. Macht der Technik. Berlin: transcript.

Jochum, Georg; Schaupp, Simon (2019): Die Steuerungswende. Zur Möglichkeit einer nachhaltigen und demokratischen Wirtschaftsplanung im digitalen Zeitalter. In: Florian Butollo und Sabine Nuss (Hg.): Marx und die Roboter. Vernetzte Produktion, Künstliche Intelligenz und lebendige Arbeit. Berlin: Dietz, S. 327-346.

Krüger, Marcel; Frey, Philipp (2020): Neutralität, Objektivität, Normativität. Elemente einer kritischen Theorie der Technikfolgenabschätzung. In: Linda Nierling und Helge Torgersen (Hg.): Die neutrale Normativität der Technikfolgenabschätzung. Konzeptionelle Auseinandersetzung und praktischer Umgang. Baden-Baden: Nomos, S. 57-76.

Kulke, Magnus; Wadephul, Christian (2020): Digitale Dystopien utopisch aufheben - durch gesellschaftliche Aneignung. In: Berliner Debatte Initial 31 (1/2020), S. 97-110.

Maasen, Sabine; Dickel, Sascha; Schneider, Christoph (Hg.) (2020): TechnoScienceSociety. Technological reconfigurations of science and society. Heidelberg: Springer Nature. DOI: 10.1007/978-3-030-43965-1.

Martens, Helmut; Scholz, Dieter (2010): Wirtschaftsdemokratie neu denken. Eine Herausforderung angesichts der neuen Weltwirtschaftskrise. Online verfügbar unter http://www.fnpa.de/content/NeueWirtschaftsdemokratie/ FNPA_Scholz_Martens_WirtschaftsdemokratieNeuDenken.pdf, zuletzt geprüft am 20.10.2020.

Naphtali, Fritz (1928/1966): Wirtschaftsdemokratie. Ihr Wesen, Weg und Ziel. Frankfurt am Main: Europäische Verlagsanstalt.

Negt, Oskar (2011): Keine Zukunft der Demokratie ohne Wirtschaftsdemokratie. In: Hartmut Meine, Michael Schumann und Hans-Jürgen Urban (Hg.): Mehr Wirtschaftsdemokratie wagen! Hamburg: VSA, S.7-13.

Quartier Zukunft et al. (Hg.) (2020): Dein Quartier und Du. Nachhaltigkeitsexperimente im Reallabor zu Nachbarschaften, Bienen, Naschbeeten, Kreativität und Konsum. Karlsruhe: KIT Scientific Publishing. DOI: 10.5445/ KSP/1000076132.

Sadowski, Jathan (2020): Too smart. How digital capitalism is extracting data, controlling our lives, and taking over the world. Cambridge (Massachusetts): The MIT Press.
Schneidewind, Uwe (2018): Die Große Transformation. Eine Einführung in die Kunst gesellschaftlichen Wandels. Frankfurt am Main: S. Fischer Verlag.

Scholz, Trebor; Schneider, Nathan (2017): Ours to hack and to own. The rise of platform cooperativism, a new vision of the future of work and a fairer internet. London: OR Books.

Schomberg, René von (2013): A vision of responsible research and innovation. In: Richard Owen, John Bessant und Maggy Heintz (Hg.): Responsible Innovation. Chichester, UK: John Wiley \& Sons, Ltd, S. 51-74.

Schröder, Julia (2019): Das Politische in der Technikfolgenabschätzung. Reflexionen mit der pluralen, radikalen Demokratietheorie von Laclau und Mouffe. In: TATuP - Zeitschrift für Technikfolgenabschätzung in Theorie und Praxis 28 (3), S. 62-67. D0I: 10.14512/tatup.28.3.62.

Slobodian, Quinn (2018): Globalists. The end of empire and the birth of neoliberalism. Cambridge (Massachusetts): Harvard University Press.

Srnicek, Nick; Williams, Alex (2016): Inventing the future. Postcapitalism and a world without work. London: Verso.

Tyfield, David; Lave, Rebecca; Randalls, Samuel; Thorpe, Charles (Hg.) (2017): The Routledge handbook of the political economy of science. London: Routledge.

Urban, Hans-Jürgen (2019): Wirtschaftsdemokratie als Transformationshebel. Was das Konzept Guter Arbeit verlangt. In: Blätter für deutsche und internationale Politik 11/2019, S. 105-119.

Vilmar, Fritz (1975): Wirtschaftsdemokratie. Theoretische und praktische Ansätze, entwickelt auf der Basis des Gewerkschaftlichen Grundsatzprogramms in der BRD. In: Fritz Vilmar (Hg.): Industrielle Demokratie in Westeuropa. Reinbek bei Hamburg: Rowohlt, S. 26-78.

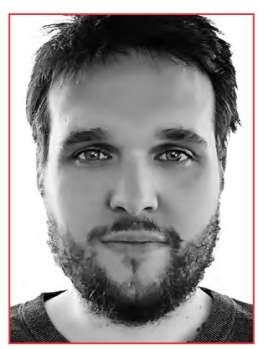

\section{PHILIPP FREY}

ist seit 2016 Doktorand am ITAS. In seiner Forschung befasst er sich unter Bezugnahme auf die frühe Frankfurter Schule schwerpunktmäßig mit der Zukunft der Arbeitsgesellschaft und Utopien der Automatisierung. Außerdem gehört er dem Vorstand des Zentrum Emanzipatorische Technikforschung an.

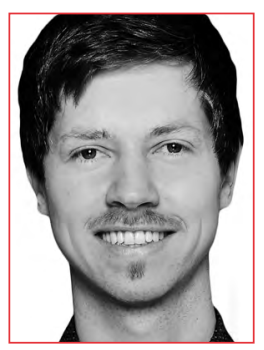

\section{CHRISTOPH SCHNEIDER}

forscht am ITAS zur Rolle von Zukunftsbildern in der politischen Ökonomie neuer digitaler Technologien und zu Möglichkeiten der Demokratisierung technischen Wandels. Er berät Organisationen zu digital-sozialer Innovation und ist in mehreren zivilgesellschaftlichen Organisationen für Demokratie und Klimaschutz aktiv.

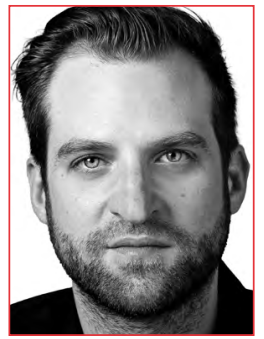

\section{CHRISTIAN WADEPHUL}

ist seit 2015 als Akademischer Mitarbeiter am ITAS in mehreren BMBF-Projekten tätig, die sich alle um die Themen Digitalisierung, Computer \& Gesellschaft drehen. Seine Forschungsschwerpunkte liegen im Bereich Technikphilosophie und -ethik, v. a. KI \& maschinelles Lernen, sowie im Bereich Automatisierung und Governance von und durch Algorithmen. 